\title{
Planetary Defense: Options for Deflection of Near Earth Objects
}

\author{
R.B. Adams ${ }^{*}$, G. Statham ${ }^{\dagger} 1,2$, R. Hopkins ${ }^{1}$, J. Chapman 1 , S. White ${ }^{1,2}$, \\ J. Bonometti ${ }^{1}$, R. Alexander ${ }^{1}$, S. Fincher ${ }^{1}$, T. Polsgrove ${ }^{*}$, and M. \\ Kalkstein ${ }^{*} 1$
}

${ }^{1}$ NASA Marshall Space Flight Center, Advanced Concepts Department, Huntsville, AL 35812, USA.
2 ERC Inc., 555 Sparkman Drive, Executive Plaza, Suite 1622, Huntsville, AL 35816, USA

Abstract. Several recent near-miss encounters with asteroids and comets have focused attention on the threat of a catastrophic impact with the Earth. This document reviews the historical impact record and current understanding of the number and location of Near Earth Objects (NEO's) to address their impact probability. Various ongoing projects intended to survey and catalog the NEO population are also reviewed.

Details are then given of an MSFC-led study, intended to develop and assess various candidate systems for protection of the Earth against NEO's. An existing program, used to model the NEO threat, was extensively modified and is presented here. Details of various analytical tools, developed to evaluate the performance of proposed technologies for protection against the NEO threat, are also presented. Trajectory tools, developed to model the outbound path a vehicle would take to intercept or rendezvous with a target asteroid or comet, are described. Also, details are given of a tool that was created to model both the un-deflected inbound path of an NEO as well as the modified, post-deflection, path.

The number of possible options available for protection against the NEO threat was too numerous for them to all be addressed within the study; instead, a representative selection were modeled and evaluated. The major output from this work was a novel process by which the relative effectiveness of different threat mitigation concepts can be evaluated during future, more detailed, studies. In addition, several new or modified mathematical models were developed to analyze various proposed protection systems. A summary of the major lessons learned during this study is presented, as are recommendations for future work.

It is hoped that this study will serve to raise the level attention about this very real threat and also demonstrate that successful defense is both possible and practicable, provided appropriate steps are taken.

\section{Introduction}

In FY2002 the revolutionary aerospace systems concepts (RASC) activity, managed from the Langley Research Center (LaRC) selected a broad range of projects for the year's activities. These projects were organized into five groups as shown in Figure 1. The Marshall Space Flight Center (MSFC) participated in two of these groups. The MSFC participation was led from TD30/Advanced Concepts. The work completed for Group 2 - Human Outer Planet Exploration is documented in another technical paper. MSFC participation in group 4 activities is documented here. Several projects were funded under group 4; however, MSFC's activities were confined to planetary body maneuvering. This paper outlines the work completed by MSFC in Planetary Body Maneuvering; full documentation of our efforts can be found in a NASA Technical Paper created by the authors ${ }^{1}$.

Work under the Planetary Body Maneuvering project was confined to defense of the Earth from collisions from asteroids and comets. Many of the technologies developed for protective maneuvering of planetary bodies are also applicable to maneuvering these bodies for resource utilization. Asteroids and comets can be maneuvered - in a careful and controlled way - close to the Earth to be mined for structural materials and water. The mass of these bodies could also anchor rotating tethers and skyhooks. Finally, such bodies

\footnotetext{
- AIAA Member

† AIAA Senior Member
} 
could be intentionally targeted to impact Mars or Venus in order to alter rotation speed and/or atmospheric composition.

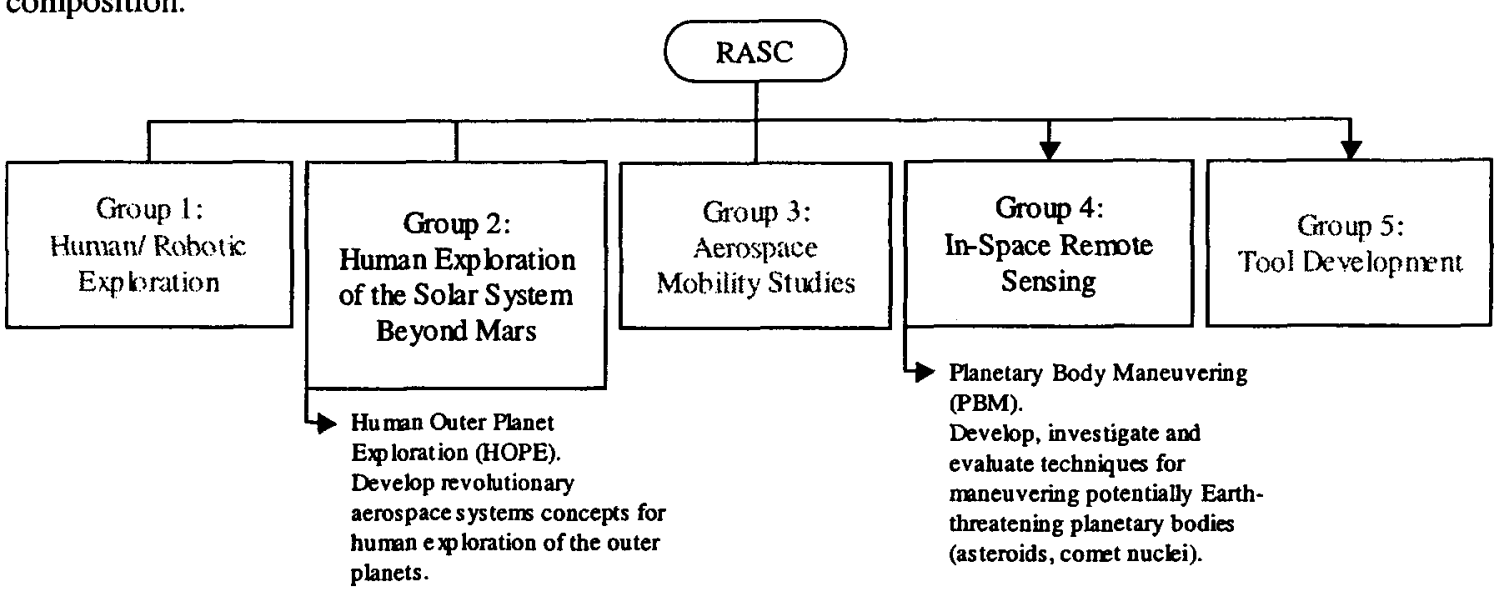

Figure 1 - Organization of RASC FY2002 activities

Despite these other potential applications for maneuvering technologies, this project concentrated solely on planetary defense as the most critical mission. We note that this mission is a uniquely suitable one for NASA. This can be seen immediately from the NASA mission statement:

\section{To understand and protect our home planet To explore the Universe and search for life To inspire the next generation of explorers ... as only NASA can}

It has been suggested that the mission of planetary defense is best suited to the Department of Defense (DoD). This argument is based on the DoD's extensive experience in the interception of high-speed objects. However, the very high-energies necessary for deflection of massive planetary bodies, combined with the unique problems of operation in interplanetary space, suggest that NASA will have a major, if not a leading role to play. The above mission statement suggests very strongly that the Agency should address this threat. NASA's unique capabilities may well make it the most uniquely qualified organization in the World to take on the daunting task of protecting the planet from this threat.

Research - conducted by the TD30 PBM team - to understand and categorize the threat of impact by an asteroid or comet is summarized on the following pages. Using the limited knowledge currently available on the solar system's asteroid and comet population, an analytical tool was developed to estimate the number of human lives that could potentially be lost because of this threat over a specified period of time. Propulsion technologies suitable for reaching the approaching object were then researched and deflection methods also investigated. Analytical tools were developed to model the actual deflection techniques. These various tools were then linked with an additional set of tools capable of modeling both inbound and outbound trajectories. Parametric results could them be generated using the linked propulsion, deflection and trajectory tools to calculate optimal deflection techniques for use against specific threat scenarios. Finally, these parametric results are presented and a set of conclusions established as to the effectiveness of each deflection method.

\section{Earth's Impact Record}

Table 1 lists the impact frequency for progressively larger Near Earth Objects (NEO's). Crater diameters and terrestrial events likely to inflict comparable damage are also listed. Note that even the smallest diameter objects are capable of causing very major damage. A $23 \mathrm{~m}$ diameter object can cause destruction 
equivalent to the nuclear weapon used at Hiroshima at the end of World War II. At the other end of the size spectrum is the Chicxulub impact, which is widely believed to have initiated an ice age at the boundary between the Cretaceous and Tertiary periods and the consequent extinction of over $50 \%$ of the then existing species of flora and fauna, including the dinosaurs.

Figure 2 illustrates the location of 145 known impact craters distributed around the World. The actual number of Earth impacts is thought to be much higher, but most of the evidence has been destroyed or covered by geological processes and vegetation. Note that the majority of the craters are less than $50 \mathrm{Ma}$ (Mega annum or million years) old. Additionally most crater diameters are in the 50-100 km range. This evidence supports the theory that wind and water erosion, seismic events, vegetation, and the like are constantly erasing crater sites. Somewhat perversely, craters above $100 \mathrm{~km}$ are not always simple to find. This is because their effects are so widespread as to not be easily recognized as a impact craters. For instance the Chicxulub crater was eventually only identified from radar density mapping performed by a petroleum company owned by the Mexican government. Sometimes there is circumstantial evidence that indicates that a major impact crater is present. In the case of Chicxulub, a large number of sinkholes were found around the periphery of the impact crater. As an interesting side note, these sinkholes contained potable water, without which it may have been impossible for the Spaniards to explore that portion of the continent in the 1700's.

Figure 2 also illustrates which parts of the Earth have been subjected to the most thorough search for evidence of extraterrestrial impacts. Many more impacts have been found in Europe, North America and Australia than in other regions. There is no obvious reason why these continents would have received a higher impact flux than the others. It seems likely that impact structures exist in equivalent numbers - but as yet undiscovered - on the other continents. Finally note that few impact structures have been found underwater. It is expected that cratering is mitigated by the cushioning effect of the oceans and that the erosion rate is higher for submerged craters. In addition, it is clearly more difficult to find craters in deep water.

Table 1 - Comparable terrestrial events for NEO's of various diameters ${ }^{2}$

\begin{tabular}{|c|c|c|c|c|}
\hline $\begin{array}{l}\text { NEO } \\
\text { Diameter }\end{array}$ & $\begin{array}{c}\text { Yield } \\
\text { (TNT } \\
\text { equivalent) }\end{array}$ & $\begin{array}{l}\text { Impact } \\
\text { Frequency } \\
\text { (per Myr) }\end{array}$ & $\begin{array}{l}\text { Crater } \\
\text { Diameter }\end{array}$ & Comparable Terrestrial Event \\
\hline $2 \mathrm{~m}$ & 500 tons & 250,000 & $35 \mathrm{~m}$ & Minimum damaging earthquake $(M=5)$ \\
\hline $4 \mathrm{~m}$ & 4,500 tons & 69,000 & $75 \mathrm{~m}$ & Largest Chemical Explosion (Heligoland Fortifications, 1947) \\
\hline $6 \mathrm{~m}$ & 20,000 tons & 28,000 & $120 \mathrm{~m}$ & Atomic Bomb Explosion (Hiroshima, Japan, 1945) \\
\hline $23 \mathrm{~m}$ & $1 \mathrm{MT}$ & 2,700 & $450 \mathrm{~m}$ & "Typicar" Hydrogen Bomb Explosion (1 MT) \\
\hline $55 \mathrm{~m}$ & $11 \mathrm{MT}$ & 540 & $1.1 \mathrm{~km}$ & $\begin{array}{l}\text { Barringer Meteor Crater, Arizona; Tunguska Explosion, } \\
\text { Siberia, Russía }\end{array}$ \\
\hline $250 \mathrm{~m}$ & $1,400 \mathrm{MT}$ & 35 & $5 \mathrm{~km}$ & Gardnos, Norway; Goat Paddock, Australla \\
\hline $500 \mathrm{~m}$ & $10,000 \mathrm{MT}$ & 10 & $10 \mathrm{~km}$ & Lake Mein, Sweden; BosumtwI, Ghana; Oasis, Llbya \\
\hline $1 \mathrm{~km}$ & $87,000 \mathrm{MT}$ & 2.9 & $20 \mathrm{~km}$ & $\begin{array}{l}\text { Haughton Dome, Canada; Rochechouart, France; Rles } \\
\text { Crater, Germany }\end{array}$ \\
\hline $1.5 \mathrm{~km}$ & $310,000 \mathrm{MT}$ & 1.4 & $31 \mathrm{~km}$ & Total annual energy release from Earth (Seismic, Volcanic, etc.) \\
\hline $10 \mathrm{~km}$ & 8.7E7 MT & 0.007 & $200 \mathrm{~km}$ & $\begin{array}{l}\text { Sudbury, Canada; Vredefort, South Africa; Chicxulub, } \\
\text { Mexico }\end{array}$ \\
\hline
\end{tabular}



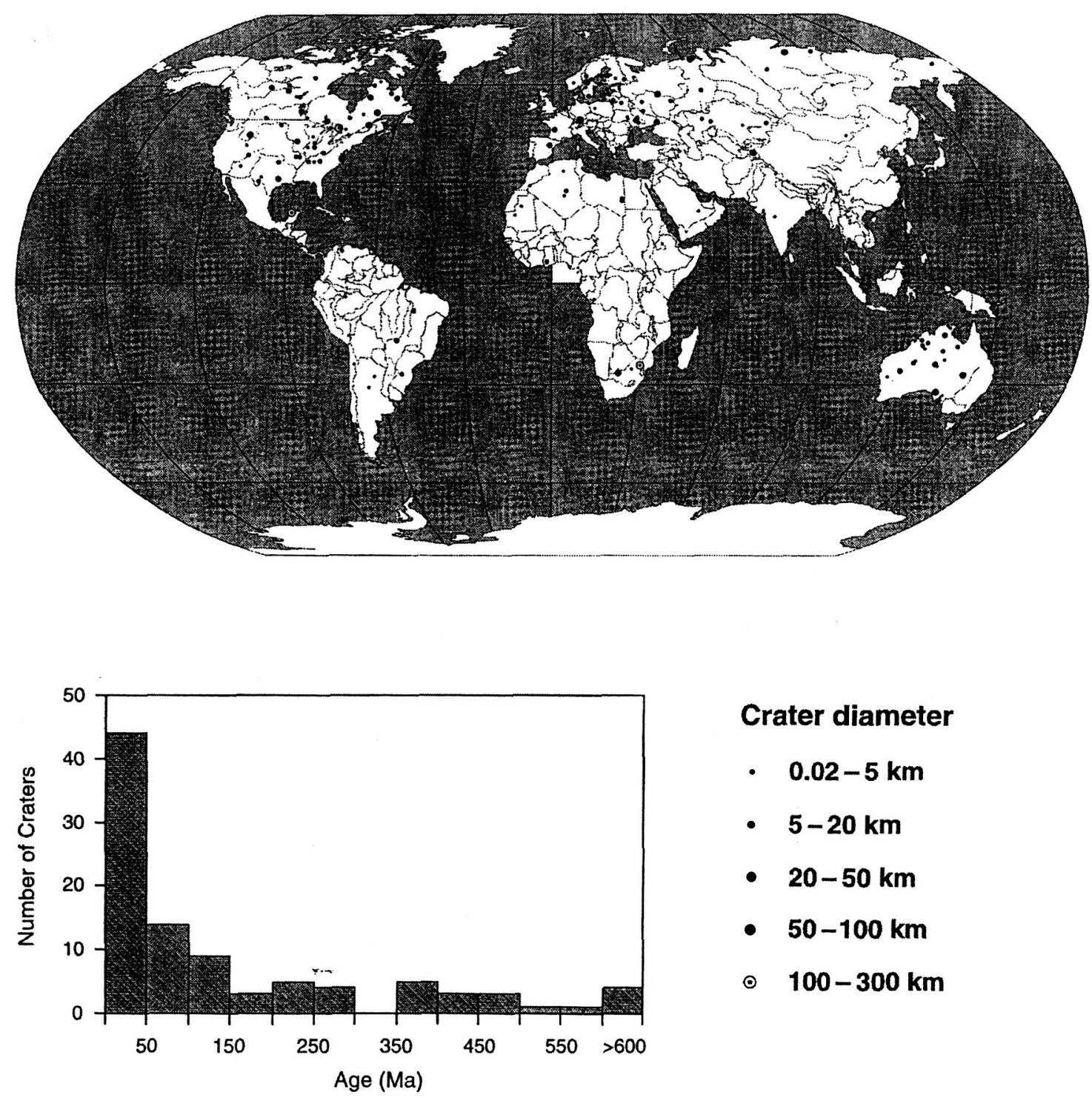

\section{Crater diameter}

- $0.02-5 \mathrm{~km}$

- 5-20 km

- $20-50 \mathrm{~km}$

- $50-100 \mathrm{~km}$

$\odot \quad 100-300 \mathrm{~km}$

Figure 2 - Location of known impact craters noting diameters as of 1998. Age distribution of these craters is also included. ${ }^{2}$

The last significant impact on Earth was the Tunguska event of 1908. This impact is believed to have been caused by a 30-60 meter object that detonated at a height of approximately $8 \mathrm{~km}$ above the surface. The estimated blast point is illustrated in Figure 3. Investigators who explored the area during a series of expeditions between 1958 and 1965 carefully recorded the directions in which trees had fallen as a result of the blast. These directions are mapped in Figure 3; they clearly indicate the location of the center of the event.

Most strikes by large NEO's do not reach the Earth's surface. Instead the combination of heat and stress which the object experiences as it travels at very high speeds through the atmosphere usually causes it to disintegrate explosively. Unfortunately, modeling and empirical evidence suggest that the heights at which such explosive blasts are most likely to occur are similar to that determined - by nuclear weapons experts to cause maximum surface damage. 
Figure 3 indicates the total ground area affected by even this relatively small object. Living creatures inside this area are not thought to have survived the event. Fortunately Tunguska is an unpopulated area in Russian Siberia. A similar strike in a populated area would have caused widespread devastation. Consider Figure 4. This is the Tunguska event superimposed over Madison County, Alabama, in the United States of America; the authors' residence and location of the Marshall Space Flight Center. An impact of this magnitude would devastate the county, killing the majority of its 250,000 inhabitants. Superimposed over a more densely populated area, such as a large city like New York or London, the devastation would cause the deaths of several million people.

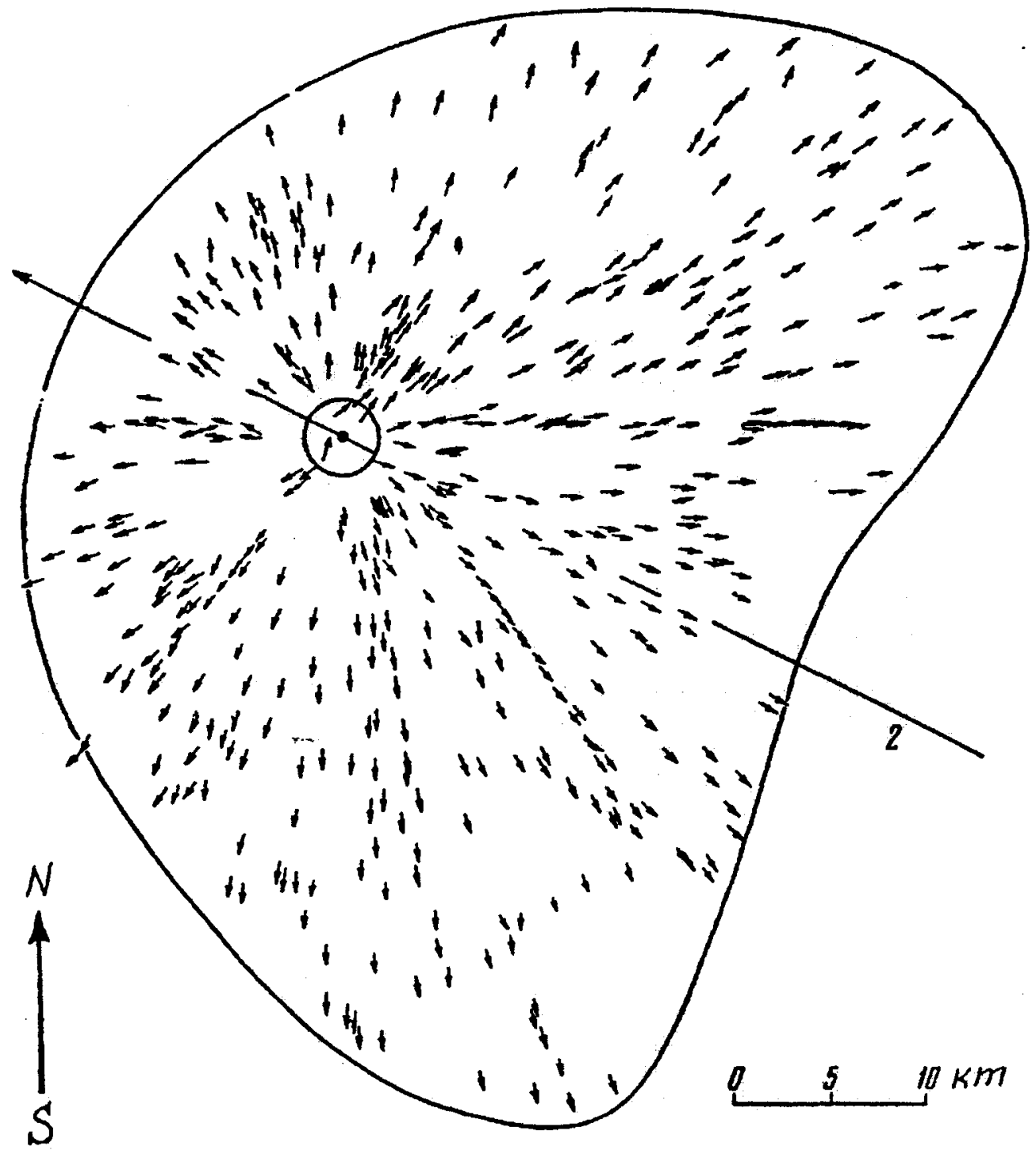

Figure 3 - Projected area affected from the Tunguska blast of 1908. Arrows depict the location and direction trees were knocked down from the blast. ${ }^{3}$ 


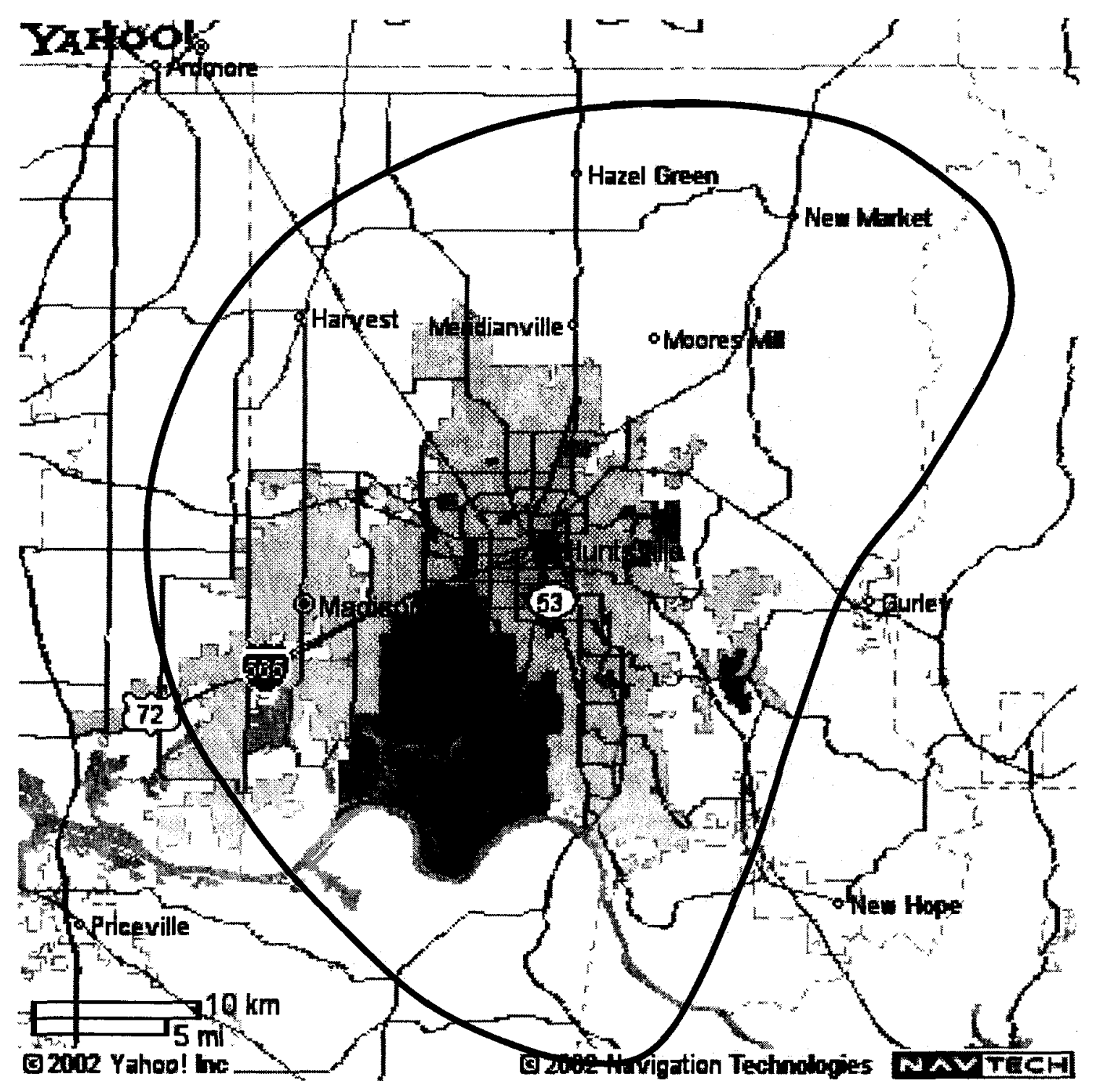

Figure 4 - Tunguska impact area superimposed over Madison County, Alabama, USA. Several hundred thousand casualties can be expected from such an impact.

\section{Measuring the NEO population}

To determine the overall threat posed by the solar system's asteroids and comets, one must develop a proper understanding of the populations of these two types of body. Unfortunately neither population is well understood. It is believed that orbital parameters are currently known for only about $10 \%$ of the total NEO population. Also, since larger objects are easier to detect, our knowledge of the known NEO population is biased towards these larger objects.

Using the known NEO population the relative location of these objects can be plotted together with the orbits of the inner planets. This plot is shown in Figure 5 and gives the location of all known objects on March 2, 2002. The green circles are minor objects that are not considered candidates for Earth impact. The red circles are minor objects that have perihelia less that $1.3 \mathrm{AU}$. Blue squares represent periodic comets. The planets are shown as crosshair circles on their orbits. The figure illustrates the large population of NEO's around the Earth at any time. The figure does create a misleading impression: due to the finite pixel size, the inner solar system - particularly the asteroid belt - appears to be full of NEO's; in fact of course, it is overwhelmingly empty. 


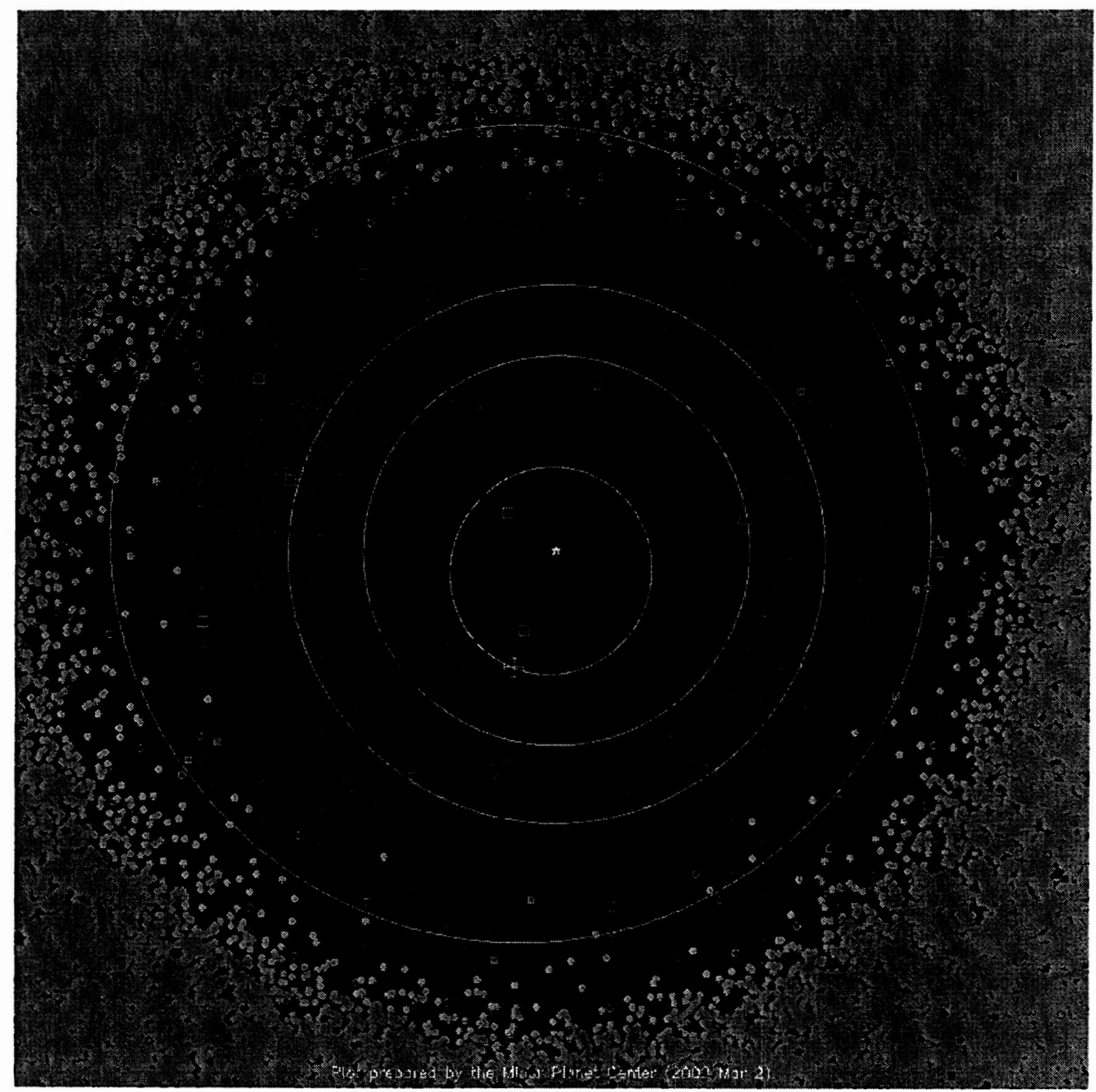

Figure 5 - Location of known minor planets on March 2, 2002 plotted relative to the inner planets. NEO's are red, other asteroids are green and comets are blue.

Our knowledge of the NEO population has increased significantly in the past few years. The number of known near Earth asteroids is plotted shown as a function time in Figure 6. The rapid increase in the number of objects identified over recent years can be explained in a number of ways. First, the recent acceptance of the Alvarez hypothesis - and the theory that other Earth impacts have also affected the planet in the past - have led to a significant increase in the time and resources available to locate these objects. Second, the development of charged coupled devices (CCD's) has computerized the previously manual process of searching for new celestial objects. Before the advent of CCD technology, such searches were conducted by the human study of photographic plates. Third, use of the Internet has facilitated international coordination and the sharing of data sharing from sky surveys.

Note that, over recent years, the total number of asteroids located has increased more rapidly than the number of large. This is due to the fact that CCD's enable the detection of smaller objects than was previously possible. 


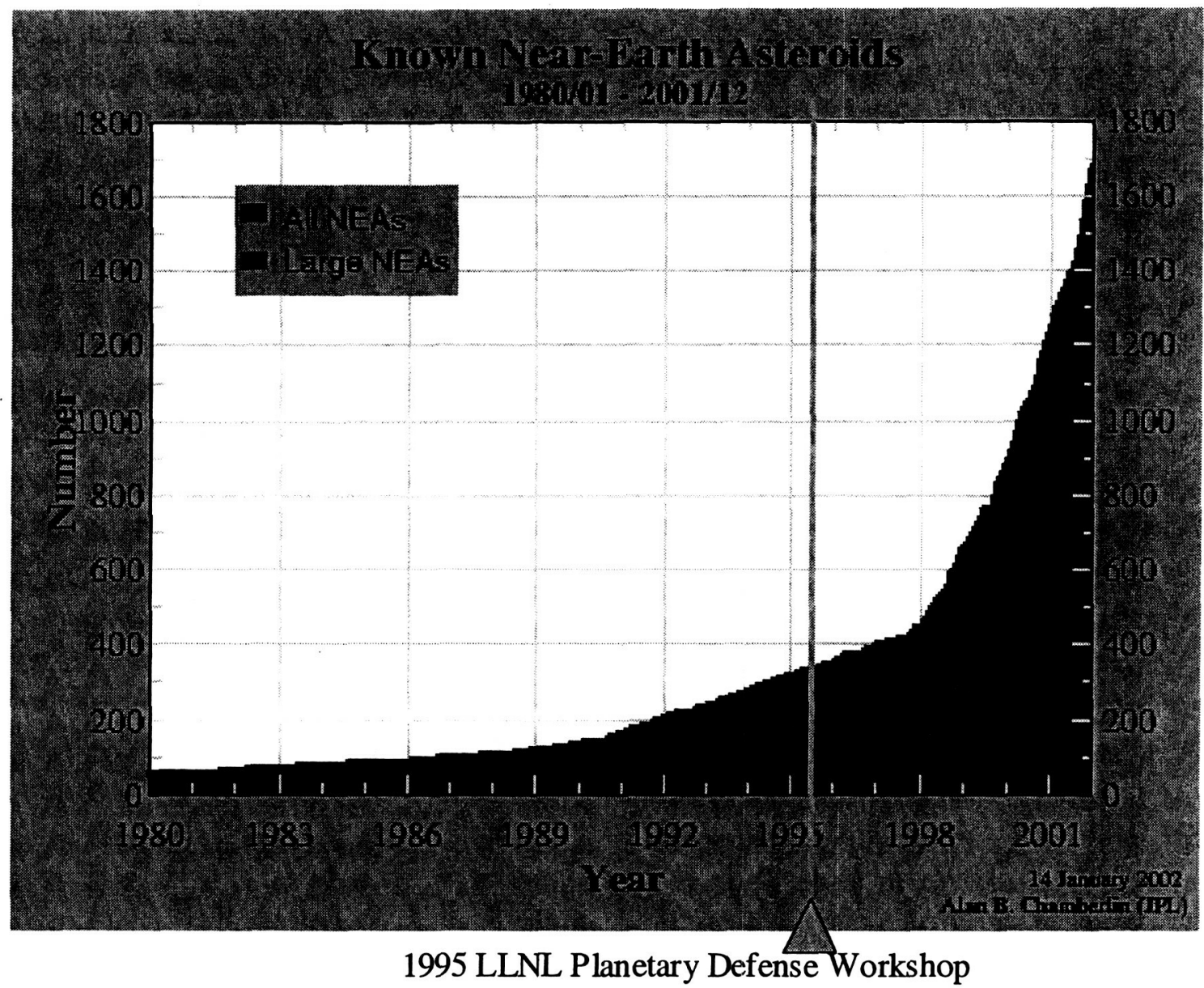

Figure 6 - Number of known near Earth asteroids vs. time. Note the rapid increase in discoveries in resent years due to the use of CCD's and increased interest in the asteroid and comet threat. ${ }^{4}$

Table 2 - Recent near misses by comets and asteroids. By comparison, the distance between the Earth and the Moon is approximately 240,000 miles

- 2001 YB5

- $\quad 300-400$ m diameter

- January 7, 2002 flew 375,000 mi from Earth

- 2002 EM7

○ $70 \mathrm{~m}$ diameter

- March 8, 2002 flew 288,000 mi from Earth

- 2002 NY40

- $800 \mathrm{~m}$ diameter

- August 18,2002 will fly $330,000 \mathrm{mi}$ from Earth

- $\quad 2002$ NT7

O $1.2 \mathrm{~km}$ diameter, $28 \mathrm{~km} / \mathrm{s}$

O Will fly by Earth on February 1, 2019

Several movies and television programs have been released in recent years giving the general public an appreciation, however scientifically distorted, of the NEO threat. The increase in NEO detection rates, coupled with the increase in public awareness, has raised the level of coverage given in the general media to this threat. Recently, major news outlets have published several articles detailing the Earth's close encounters with NEO's; the list below gives some details. To our knowledge, several asteroids have passed 
the Earth at a distance of less than twice the Moon's orbital radius from the Earth in the past year. In at least one instance the detection was made after the asteroid had already passed the point of closest approach.

\section{Mission Configurations}

In considering how to counter an incoming object it is important to consider first whether it is better to push the object out of the way or to break it up into small pieces. Each option offers its own set of advantages and disadvantages. Additionally one must consider how to deliver the energy needed to deflect or fragment the object. Three methods are discussed here: remote station, interception and rendezvous. Under the remote station approach, no complex spacecraft would be sent out to the approaching NEO; instead all operations are conducted remotely - probably from the vicinity of the Earth - with beamed energy or projectiles being used to perform the deflection or fragmentation. A strategy based upon interception would involve sending spacecraft out on a intercept trajectory with the incoming NEO; the resulting high velocity impact(s) would accomplish either deflection or fragmentation. Rendezvous-based techniques are more propulsively demanding, as they require one to dispatch hardware to actually match orbits with the incoming NEO.

At first consideration it would seem that the decision over deflection versus fragmentation is interlinked with the method chosen. An intercepting object would deliver all of its energy at once, tending to cause fragmentation instead of deflection. However, an incoming NEO could be deflected by a series of intercepting objects, each imparting enough momentum to slightly perturb its orbit without causing fragmentation. Similarly, it may seem improbable to actually rendezvous with an incoming object only to subsequently break it up however, if there were a finite amount of time needed for the system to deliver the fragmentation energy then rendezvous becomes necessary.

\section{Deflection vs. Fragmentation}

Figure 7 illustrates the concept of deflecting an incoming object away from an orbit that intersects with the Earth. In the case illustrated, it is anticipated that the deflection mechanism would require a significant period of the NEO orbit to deliver the energy necessary to perturb its orbit. The figure shows the commencement of deflection before aphelion, if undeflected, the object would collide with the Earth near perihelion.

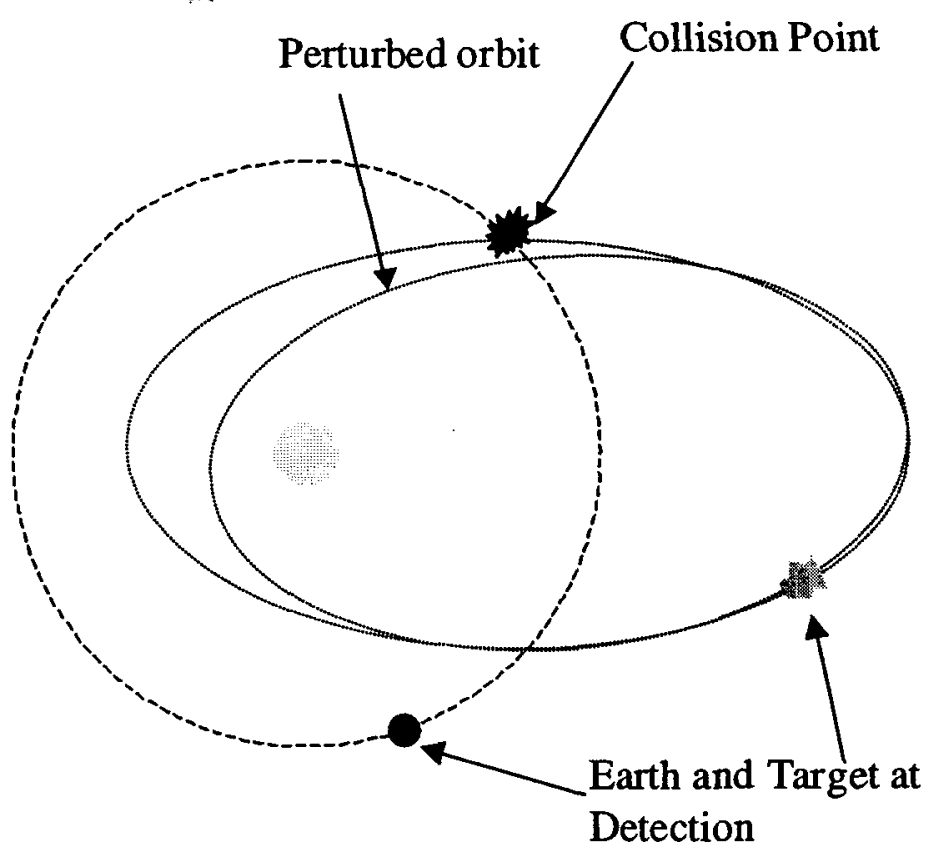

Figure 7 - Illustration of deflection method of threat mitigation 
When considering strategies based on deflection, it is important to establish what level of perturbation is necessary to consider the Earth as being safe from collision. One might argue that "a miss is good as a mile" but some margin of error is necessary when designing a system that would deflect incoming objects. The literature commonly uses a figure of 3 Earth radii as a minimum safe approach distance for a deflected object. This value takes into account the uncertainty in astrodynamical constants that affects trajectorymodeling accuracy for the incoming object.

Figure 8 illustrates the concept of fragmentation. At first sight, this might seem the best approach as the object's destruction means that it cannot threaten the Earth on a later orbit. In addition, there is no requirement to deliver the energy to the NEO in a distributed manner, thus it can be defeated in one shot. Finally, as recent Hollywood blockbuster movies clearly demonstrate, there is a unique emotional satisfaction to be derived from destroying a life-threatening object in this emphatic manner.

Despite its immediate 'tabloid' appeal, fragmentation does introduce several issues that, on reflection, make it less attractive than deflection. It is important to break the object up into relatively small components. To break the object up into just a few pieces could actually exacerbate the damage to the Earth, with several distributed impacts occurring instead of one large impact. The fragmented pieces can 'draft' off one another in the atmosphere (i.e. following pieces can travel within the slipstream of a leading piece and thus reach the ground relatively intact) alleviating burnup. For these reasons the suggested fragmentation criteria is that no fragment should have a diameter greater than $\mathbf{1 0}$ meters. A major problem arises because asteroids and comets are suspected to have very heterogeneous composition with significant internal structural flaws. Energy deposited into such objects cannot be expected to cause uniform fragmentation.

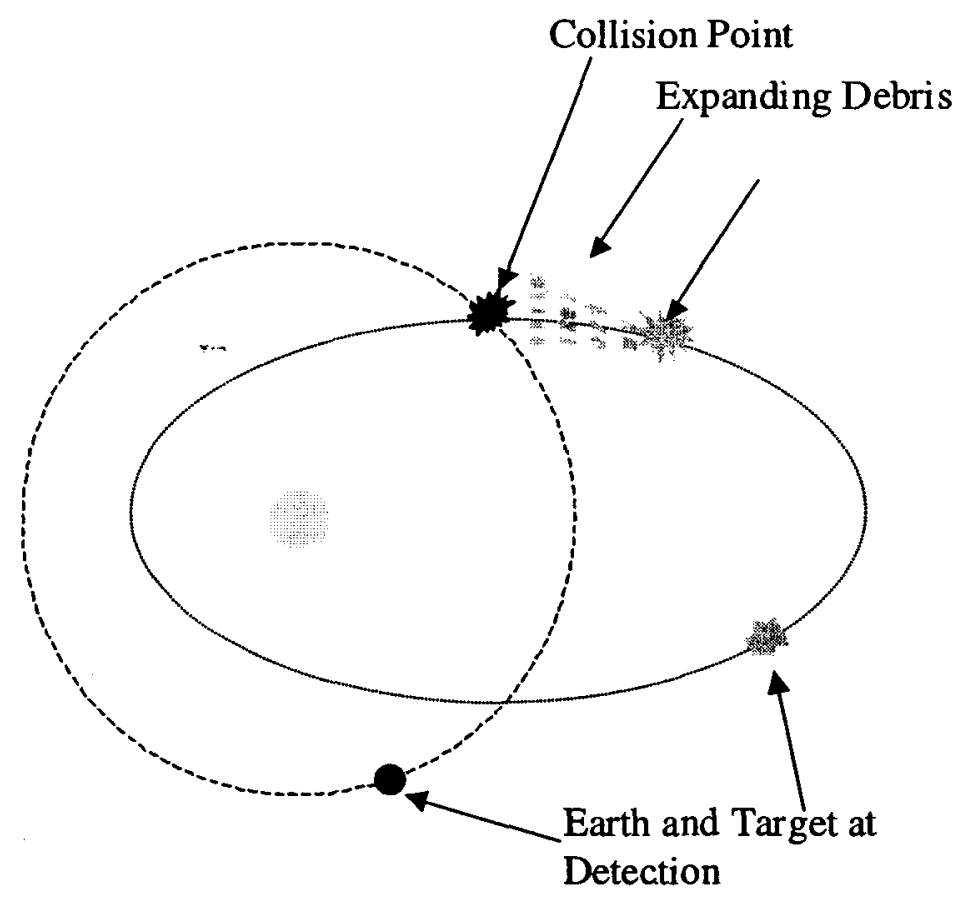

Figure 8 - Illustration of fragmentation method of threat mitigation

\section{Remote Station vs. Interception vs. Rendezvous}

The three modes considered in this study to deliver deflection or fragmentation energy to the incoming object are remote station, interception and rendezvous. The remote station mode is depicted in Figure 9. A station remains in orbit around either the Earth or the Sun. Energy can be delivered in form of projectiles fired from the station by a mass driver or by a focused beam of coherent light such as a solar lens or a laser. The advantage of such a system is that it remains close to the Earth and is easily maintained and upgraded. 
Also the system can start deflecting the incoming object almost immediately, without the delay - which might be months or years - during which an interceptor or rendezvous system would take to reach the object. However there are also several disadvantages. Targeting of the beam or stream of projectiles is a not a trivial issue. For instance targeting is required to within $1.4 \cdot 10^{-5}$ to $2.8 \cdot 10^{-6}$ arc seconds for objects between 1 and $5 \mathrm{AU}$ (the approximate orbital radii of Earth and Jupiter). Focusing the beam on the object across such vast distances would also be very challenging. There would be no vehicle in the vicinity of the object that could accurately assess the effect of the beam; any such assessment would have to be conducted remotely from terrestrial or Earth orbiting platforms. This need for remote sensing, over large distances, would make it more difficult to properly assess the effect on the NEO. Moreover, unless the station is placed in a polar orbit the object will almost certainly be eclipsed once per revolution. Polar orbits would require additional launches to deliver the station into orbit and would result in higher radiation exposure. Finally a remote station would only be able to deflect incoming objects radially away from the station. Over a finite time period, the station and object will move relative to one another, causing the deflection vector to rotate and thereby wasting some of the beamed energy. Also, one must remember that radial deflection may not be the most efficient deflection strategy.

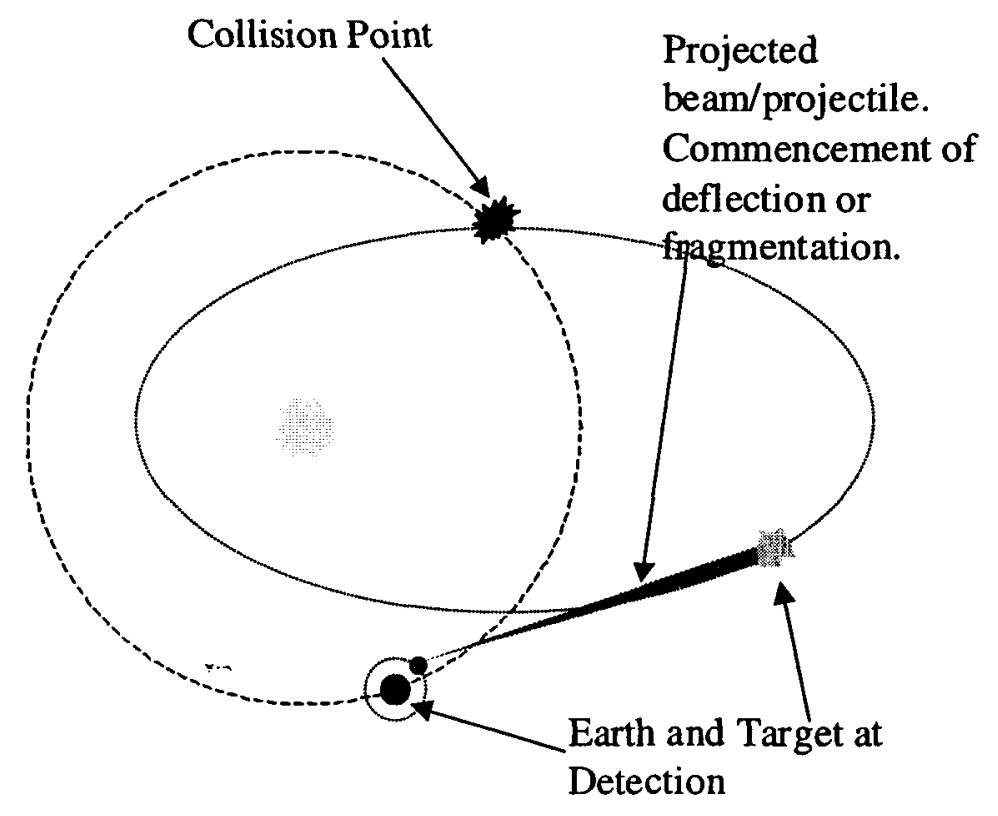

Figure 9 - Delivering deflection or fragmentation energy by the remote station mode

The interception strategy is depicted in Figure 10. After NEO detection, the interceptor is deployed to intersect it later in its orbit. At this point, deflection or fragmentation can commence. In most cases the interceptor will have substantial kinetic energy relative to the NEO. Thus the interception option allows use of some of energy initially stored in the outbound propulsion system to be delivered to the NEO. Interception options tend to be relatively simple, capitalizing on the high kinetic energy that is naturally available. The propulsive requirements for interception are substantially less than for rendezvous. This difference is further discussed in Section V: Trajectory Options.

Despite these advantages, the interception strategy also has its problems. The rate of closure between the interceptor and NEO can be as high as several tens of miles per second. This is an order of magnitude higher than the closure rate required for the kinetic kill vehicles used in the US Global Missile Defense (GMD) program. The GMD program has had a mixed success rate in interception tests against simulated Inter-Continental Ballistic Missiles (ICBM) warheads. While it is true that an incoming asteroid or comet will not maneuver to avoid destruction and, at $10-1000 \mathrm{~m}$ in size, offers a larger target than a 3-5 $\mathrm{m} \mathrm{ICBM}$, the very high closure speeds still pose significant problems for guidance and terminal maneuvering. 
Clearly only a system with multiple interceptors, dispatched in sequence, with the later vehicles capable of adjusting their trajectories, can provide the opportunity to continually sense changes in the NEO orbit. It should be noted that the interception strategy has the same type of deflection vector limitations as for the remote station strategy.

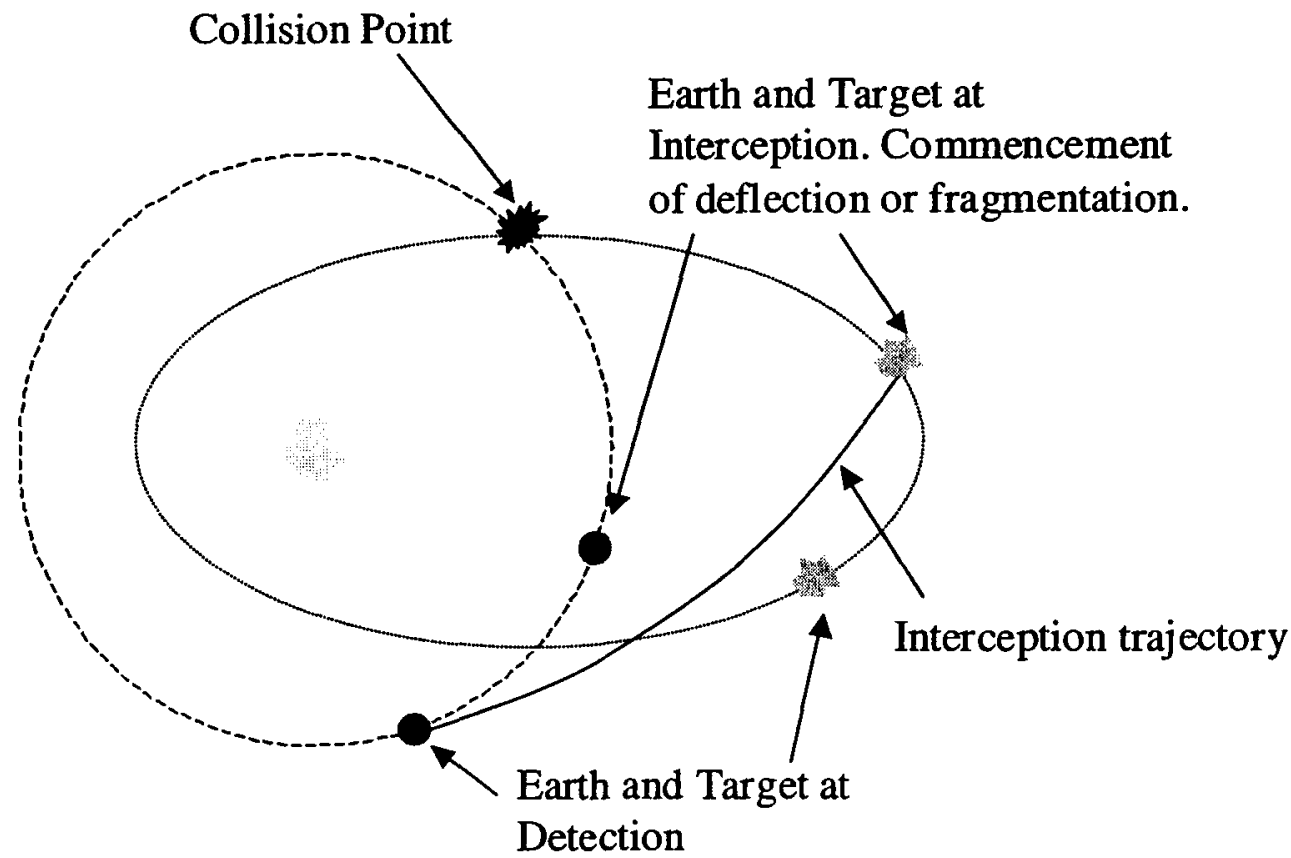

Figure 10 - Delivering deflection or fragmentation energy by the interception mode

The final strategy is that of rendezvous with the incoming NEO, as shown in Figure 11. After detection the rendezvous system is deployed and matches orbits with the NEO later along its trajectory. This strategy is the one required for most types of deflection systems. It has several significant advantages. Targeting the NEO is much less difficult for a vehicle in a parallel orbit. This strategy offers the best opportunity to continuously evaluate the NEO object during deflection or fragmentation operations. The limitations placed on the direction of the deflection vector that were encountered with the other two strategies are absent for the rendezvous option, allowing deflection in the direction that requires the least energy. Finally this strategy has the greatest synergy with resource utilization missions.

Of course the propulsive requirements to rendezvous with an incoming NEO are much higher than that for interception. Additionally the response time for a rendezvous system must include both the outbound rendezvous time and whatever inbound time is needed for the fragmentation or deflection process to take place. The rendezvous vehicle may be sufficiently distant from the Earth to make teleoperations difficult and would thus require significant on-board autonomy in an unknown environment that offers many opportunities for unexpected effects. Finally, during fragmentation or deflection the rendezvous vehicle will probably be exposed to a hazardous environment filled with ejecta from the asteroid. The vehicle will have to be designed to resist this environment. 


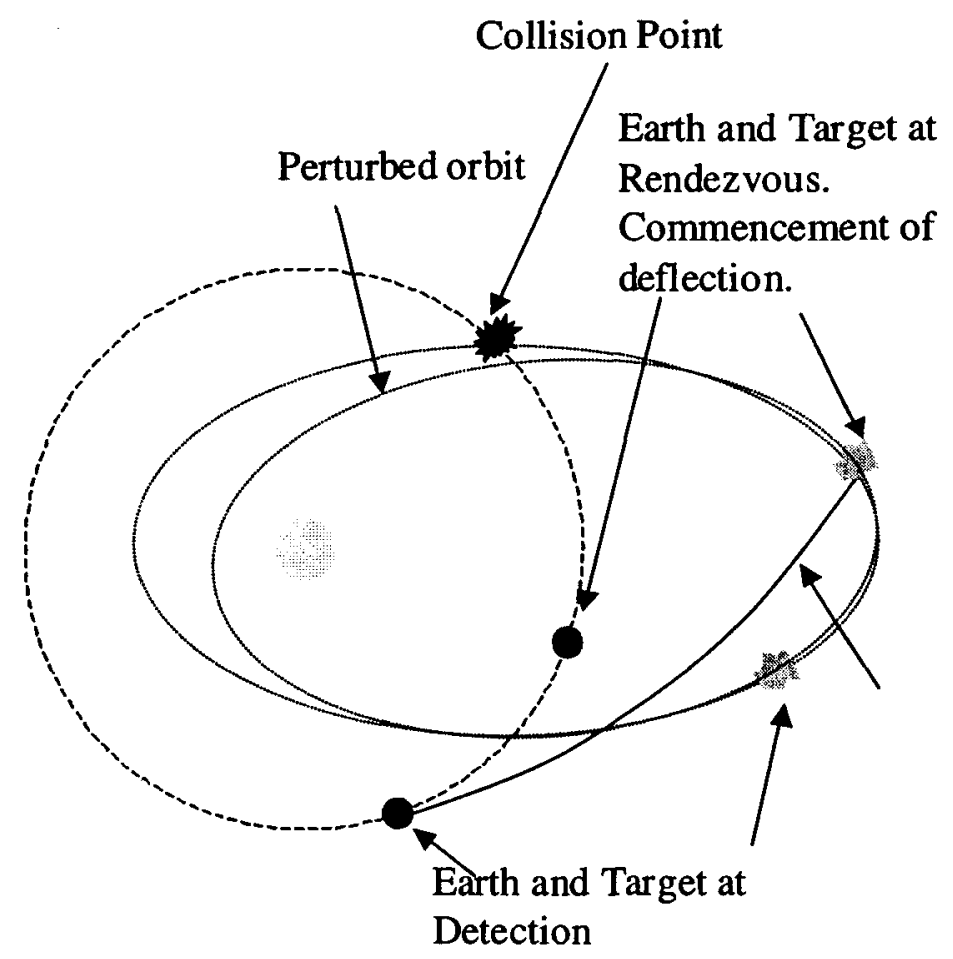

Figure 11 - Delivering deflection or fragmentation energy by the rendezvous mode

\section{Outbound Propulsion}

For both the interception and rendezvous techniques, neither fragmentation nor deflection can take place until the necessary system hardware is transported out to the approaching NEO. Some type of outbound propulsion system is required to accomplish this. Several propulsion systems are considered in this study; they were selected for their ability to meet the mission requirements, their level of technological maturity and development status. The list of outbound propulsion technologies considered in this study is listed in Table 3. It is by no means a comprehensive list but represents the interests and knowledge of the study participants. These technologies were extensively modeled and are documented in a NASA technical paper by the same authors ${ }^{1}$.

Table 3 - Outbound propulsion technologies considered in this study

\begin{tabular}{|l|}
\hline Outbound Propulsion Technologies \\
\hline Staged Chemical \\
\hline Nuclear Thermal Rocket (NTR) \\
\hline Nuclear Pulse \\
\hline Solar Sail \\
\hline Solar Collector \\
\hline
\end{tabular}

\section{Threat Mitigation}

As mentioned in the previous section, threat mitigation can occur from a remote station beaming or firing projectiles at the incoming object, or can be produced from a vehicle on an intercept or rendezvous trajectory with the object. In each case there are several options for delivering the required deflection energy. Threat mitigation techniques use kinetic, photonic and nuclear energy, to name a few. Table 4lists the threat mitigation techniques that were investigated in this study. As mentioned above these techniques were selected mainly due to the study members interest and knowledge and should by no means be 
considered a complete list. These technologies are also documented in detail in our NASA technical paper $^{\text {Error! Bookmark not defined. }}$

Table 4 - Threat mitigation technologies considered in this study

\begin{tabular}{|l|}
\hline Threat Mitigation Technologies \\
\hline Nuclear Fragmentation \\
\hline Nuclear Deflection \\
\hline Solar Sail \\
\hline Solar Collector \\
\hline Magnetic Flux Compression \\
\hline Mass Driver \\
\hline Kinetic Deflection \\
\hline
\end{tabular}

\section{Trajectory Modeling}

\section{Outbound}

The outbound trajectory was modeled simply using two-body orbital mechanics and impulsive thrust assumptions. These assumptions are not accurate for the continuous thrust propulsion systems considered, but the use of a more accurate integrating trajectory optimization program would have required more time than was available for this project. These inaccuracies must be considered in any follow-on study.

The outbound trajectory is solved as a Gauss problem. Two points in space are known, as well as the desired transfer time between them. The asteroid's position at interception or rendezvous is calculated by assuming an Earth impact position and traveling backwards, along the asteroid's orbit, to the desired arrival time, which is given as the number of days before impact. In this study, the asteroid's orbit is initialized such that it will impact the Earth at a 45-degree angle on the heliocentric-ecliptic plane. The spacecraft's position at departure is the same as the Earth's at that time. The Earth's position is calculated by moving the Earth backwards from the impact point by the number of days equal to the asteroid arrival time (given as the number of days before impact) plus the desired outbound trajectory flight time.

The Gauss problem formulation used in this study is taken from the literature ${ }^{5}$. The universal variables solution method allows the trajectory to be any type of conic section: an ellipse, a parabola, or a hyperbola. Two $\Delta V$ s are calculated. The first must be applied to depart Earth's orbit and send the vehicle on the trajectory that will intercept the asteroid in the desired flight time. The second is applied upon arrival at the asteroid. This $\Delta \mathrm{V}$ places the vehicle in the asteroid's orbit and allows for rendezvous with the asteroid. Interceptor missions like kinetic deflection use only the first $\Delta \mathrm{V}$ since impact with the asteroid is desired. Other missions that require close asteroid operations must perform both maneuvers.

\section{Inbound}

The inbound trajectory modeling software determines the minimum impulsive $\Delta \mathrm{V}$ required to make an incoming planetary body miss Earth by some specified distance. In order to allow the inclusion of other objects - such as Jupiter - during later studies, the software numerically integrates the equations of motion of the Sun-Earth-planetary body system. The program, named PBI (Planetary Body Intercept), iterates over the search space until a $\Delta \mathrm{V}$ is found which is a minimum and also causes the planetary body to miss the surface of Earth by 3 Earth radii.

To determine minimum $\Delta \mathrm{V}$ requirements, it was necessary to find a planetary body that would definitely collide with Earth, preferably dead center. However, due to the uncertainty in the orbital determination of the Near Earth Objects (NEO), it was decided not to conduct a lengthy search of the NEO catalog. Instead, a fictitious body was created in the following way. First, the orbital elements of 444 known potentially hazardous asteroids (PHA's) were examined in order to establish those elements that might apply to an 'average' PHA. Next, the PHA database was searched for one asteroid that came close to this average - at least in terms of orbital size, eccentricity, and inclination. The resulting candidate asteroid was 1999JT6. 
Its orbital elements were then modified slightly, so as to force a collision with Earth. For this purpose, the Earth was is placed 45 degrees from the X-axis of the Heliocentric-Ecliptic coordinate system at the time of the hypothetical impact. The original and modified elements of 1999JT6 are given below in Table 5. The modified asteroid orbit is plotted in Figure 12.

Table 5 - Original and Modified Orbital Elements of 1999JT6

\begin{tabular}{lll} 
Name & $\begin{array}{l}\text { Original } \\
\text { 1999JT6 }\end{array}$ & Modified \\
Semi major axis $(\mathrm{km})$ & 319280491.2 & M1999JT6 \\
Eccentricity & 0.579033744 & 319285502.2 \\
Inclination* & 9.568048 & 0.5791277 \\
Longitude of ascending & 79.06928886 & 11.47182 \\
node* & & 45.009263 \\
Argument of Perihelion* & 38.8692997 & \\
\multicolumn{2}{c}{ *All angles are in degrees } & 41.840244
\end{tabular}

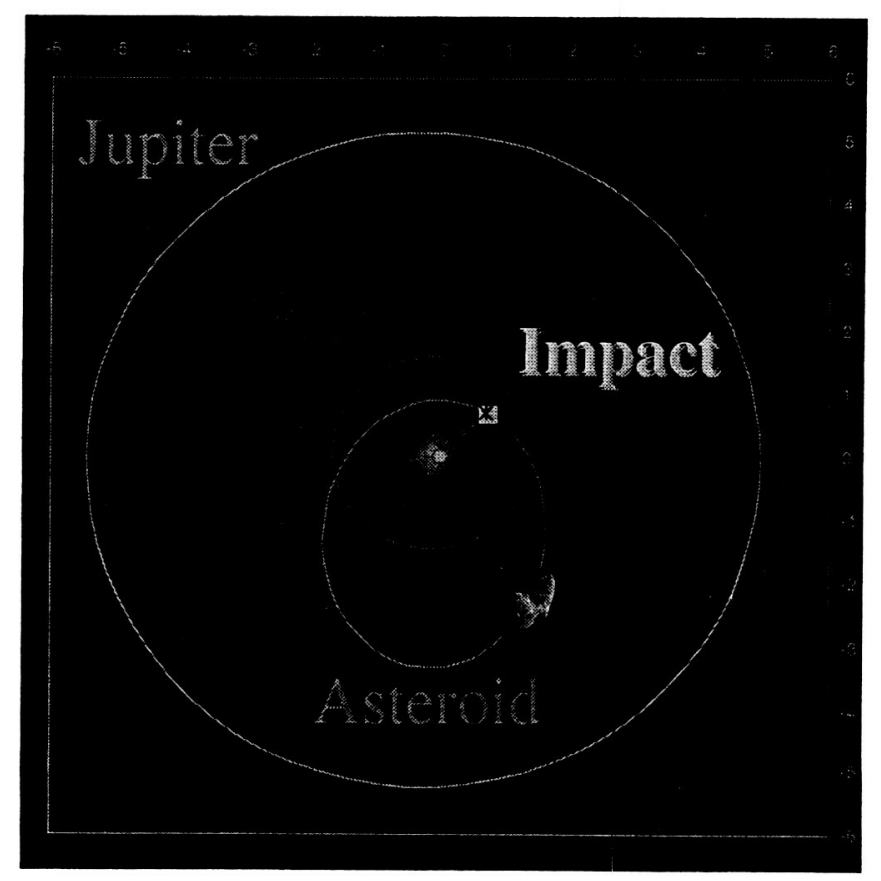

Figure 12 - M199JT6 Orbit Plot

To avoid confusion with the real asteroid 1999JT6, this fictitious asteroid has been named M1999JT6. This step was taken largely to avoid the possibility of readers getting the impression that 1999JT6 is indeed on a collision course with the earth. Size and composition of the fictitious asteroid are not specified since these characteristics are varied during the analysis. During execution of the PBI program, the user specifies only the mass and velocity vector of an arbitrary planetary body, which is initially placed at the center of the earth. Then the position of the body is adjusted so that it collides with the surface of the earth nearly dead center. For M1999JT6, the mass is varied, but the impact velocity vector is always as given below.

$$
\mathbf{V}=-35.65 \mathbf{I}+18.5 \mathbf{J}+9.0 \mathrm{~K} \quad \frac{\mathrm{km}}{\mathrm{s}}
$$


Thanks to the pioneering work of researchers like Eugene Shoemaker (1983), the threat and consequences of an impact with a planetary body are now more appreciated, and better understood, than was the case thirty years ago. One only has to consider the literature (Chapman and Morrison, 1994 ${ }^{6}$; Gold, 19997; John Lewis, $1999^{8}$; Jeffers, et al, $2001^{9}$; Chesley, $2001^{10}$; Ivezic, et al, 2001 ${ }^{11}$; and many others) to see evidence of increased efforts to understand the threat that Earth faces every day. Although our understanding of the impact threat is still incomplete, it is far ahead of our understanding of the consequences of the most likely impacts. Attempting to predict the number of people killed over the next decade, century, or millennium due to impacts of certain sizes of planetary bodies is a highly speculative endeavor. Recorded impacts in the developed world are rare and so do not constitute a statistically significant database. This means that researchers must resort to the use of theoretical models ${ }^{8}$ to estimate the number of deaths resulting from the impact of a planetary body of a certain size, velocity, and type.

Our program, which has been named "PEOPLE", estimates the number of people saved over the next century if all planetary bodies of a given type (i.e., chondrite, long period comet, etc.), having kinetic energy less than or equal to some given value, can be successfully deflected. The number of fatalities prevented is based on the work done by Shoemaker $(1984)^{12}$, Chapman and Morrison (1994) ${ }^{6}$, and Lewis $(1999)^{8}$, using estimates of both the impact frequency and the number of deaths due to impacts of a certain energy. Over 10,000 runs of a modified ${ }^{*}$ version of John Lewis's Monte Carlo simulation program were used to generate data for the average number of deaths due to each type of object. These types are listed in Table 6.

Table 6. The Types of Planetary Bodies Examined in the Monte Carlo Simulation and Their Average Contribution to the Total Number of Deaths Over the Next Century.

$\begin{array}{lc}\text { Type } & \% \text { of total deaths } \\ \text { Chondrite } & 60 \\ \text { Achondrite } & 5 \\ \text { Iron } & 5 \\ \text { Mesosiderite } & <1 \\ \text { Pallasite } & <1 \\ \text { Comet, short period } & 6 \\ \text { Comet, long period } & 24\end{array}$

The program PEOPLE also determines which parameter (mass or velocity) should be increased to counter the largest portion of the threat. This determination is based on velocity distributions from Lewis (1999) ${ }^{8}$ and Chesley $(2001)^{10}$, and size distributions from Gold (1999) ${ }^{7}$ and Ivezic, et al (2001) $)^{11 *}$. A static human population of 6 billion is assumed here, as it is throughout most of the literature. Causes of death include tsunamis, blast waves, firestorms, and direct impacts.

\section{Parametric Results}

\section{Integrated Analysis}

The original intent of this project was to evaluate the ability of various combinations of technologies to defeat the entire threat posed by NEO's. Obviously this is a very complicated problem. Potential impactors come in all shapes and sizes, and their orbits vary greatly. To further complicate matters, we have examined a large number of technologies for use in threat mitigation. Assessing all possible technology combinations would be prohibitive.

\footnotetext{
- The main modification to Lewis's simulation code allowed the tallying of deaths due to different types of objects, enabling the study team to focus efforts on the types of objects that would most likely cause the most deaths over the next century.

* Recent data from Brown, et al, appearing in the 21 November 2002 edition of Nature, indicates that the frequency for Tunguska-sized events may occur only every 1000 years as opposed to previous estimates of every 200 to 300 years, and that the size distribution of the smaller asteroids may need to be reassessed.
} 
The original intent of this project was to select several technologies based on our understanding and experience, and to test the ability of each to defeat the total threat. Figure 13 illustrates the proposed analysis process. Starting with an assumption for the total system mass and the total mission time allowed, the analysis process then divides based on the type of threat mitigation concept being considered: remote station, interception or deflection. The remote station analysis path assumes both an incoming asteroid mass and a velocity vector. Running the inbound parametric defines the $\square \mathrm{V}$ required to deflect the asteroid - given its size and velocity vector at point of impact - and also the allowable mission time. Running the remote station tool based on the $\square \mathrm{V}$ to be delivered to the object, and the allowable mission time, computes the required remote station mass. If this mass is not equal to the allowable mass for the system assumed at the beginning, then the analysis path returns to assume a new asteroid mass and velocity vector. New $\square$ V's and remote station masses are then computed. The new asteroid mass and velocity vector is selected using the threat assessment tool to maximize the percentage of the total threat that can be defeated for the assumed total system mass. After closure, the threat assessment tool is run again in order to compute the total threat that is defeated. The total threat is quantified by the percentage of people saved by deployment of the system over a given time period, divided by the number of people expected to die from impact of an NEO over the same period. By running through this process several times, assuming new total system masses and mission times on each occasion, yields a parametric model of the total threat defeated as a function of total system mass, for lines of constant mission time.

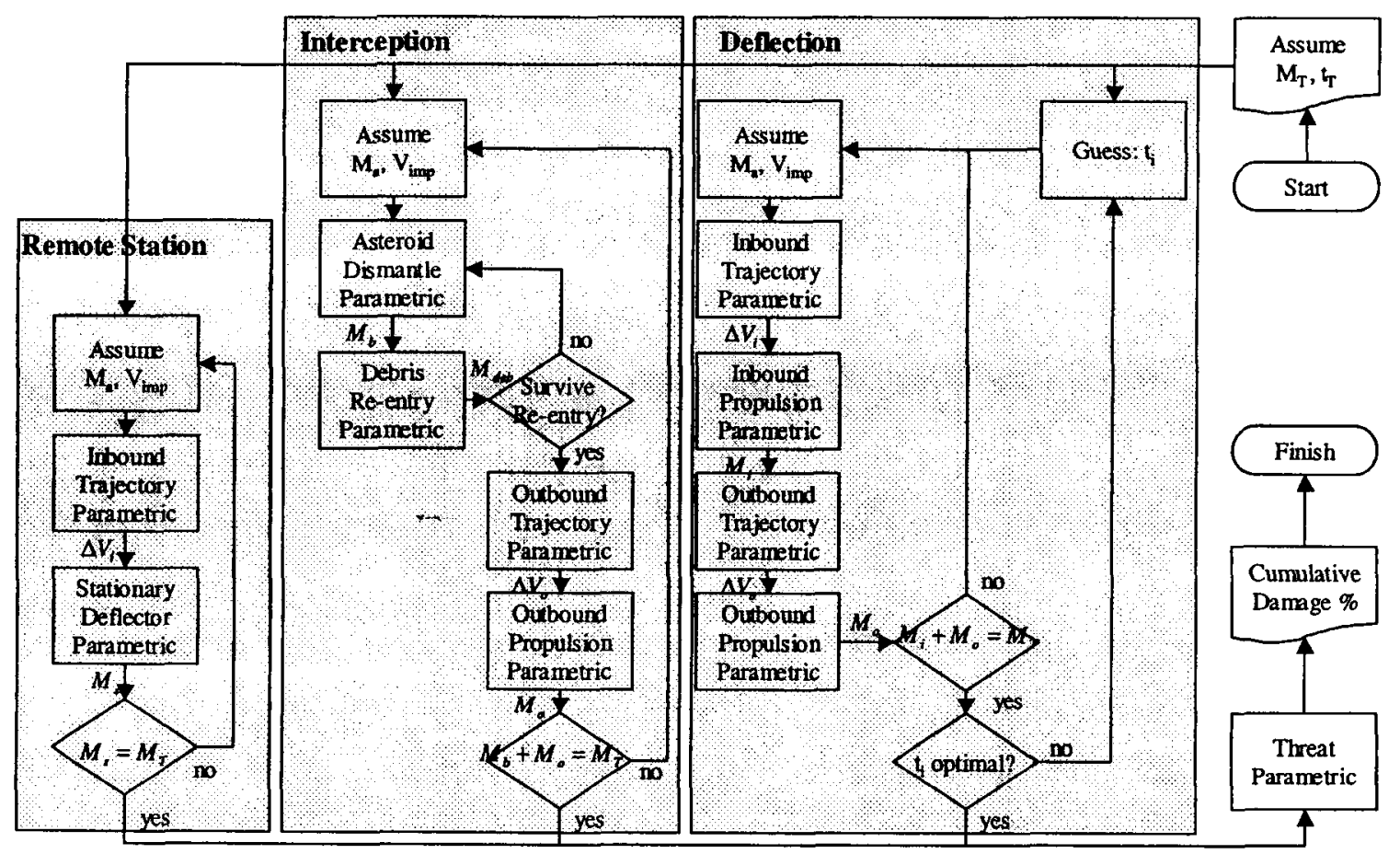

Figure 13 - Proposed analysis process for assessing total amount of threat mitigated.

The interception branch differs from that for the remote station in that the inner iterative loop determines the asteroid size and velocity that can be deflected. Here there is an additional problem in optimizing the amount of the total mission mass allocated to the outbound propulsion as against the amount allocated to the interception system. First, an interceptor mass is assumed and the size of the resulting object fragments is estimated. Development of an atmospheric entry code - that would model the burn-up of these fragments - was initiated, but this has not yet been completed. It is intended that this model would include the effects of "drafting" - that is later objects following in the wake of the earlier objects. If the fragments survived re-entry then a larger interception mass is assumed and a new iteration ensues. Otherwise the calculation proceeds to calculate the $\square \mathrm{V}$ required and then the mass of the outbound propulsion stage needed to take the interceptor to the incoming object. This outer loop iterates until the maximum threatening object that can be mitigated for the total allowable mission mass is found, again using the threat 
tool. As before, a parametric model, giving the percentage of the total threat defeated as a function of both total system mass and mission time, can be generated.

The rendezvous branch in Figure 13 is the most complicated one of all. Here the most threatening object must be found using techniques similar to those employed for the interception branch. However, in this case, an allowed inbound trip time is also assumed. The analysis process then runs through the tools that calculate the required inbound $\square \mathrm{V}$ and mass, as well as the outbound $\square \mathrm{V}$ and mass. Then the total mass can be calculated by summing the inbound and outbound masses and comparing the result with the assumed total system mass. Even after closure of this inner loop, the interception point defined by the allowed inbound interception time may not be optimal. Therefore another loop is used, to find the optimal interception point. After closure of this loop, the process goes into the threat parametric to find the total percentage of the threat that is defeated. Again a parametric model of total threat defeated as a function of system mass and mission time is generated.

There are still two assumptions built into the above analysis scheme. First, although the distribution of object mass and velocity is taken into account, the possible distribution in composition is not. We believe that composition is a secondary factor in performance - although not in damage caused. In addition, because asteroid and comet compositions are so poorly understood, it was decided that the whole issue of composition would not be addressed for this initial study. Note that the inclination distribution was taken into account - it is imposed by a non-zero $Z$ component in the incoming object velocity vector at impact.

Second, the deflection study allowed no time for setup after asteroid rendezvous. None of the options we considered required a significant amount of time after rendezvous for these operations, so it was assumed that the required deflection $\square \mathrm{V}$ was imposed instantaneously upon rendezvous. Finally, the threat parametric has several implied assumptions defined in the last section.

Unfortunately we were not able to complete these ambitious analysis goals in the time available. As will be shown below, we were able to derive a parametric model of total system mass for several architecture options, assuming a standard set of orbital elements for the incoming object. The parametrics were derived using a process similar to the inner loops shown in Figure 13. The architecture options considered are described below in part $b$. The performance of these options is also shown below.

\section{CONCLUSIONS AND RECOMMENDATIONS}

In this study we have modeled a wide range of potential mitigation techniques by which threatening planetary bodies could be either deflected or fragmented. We have also considered a range of transportation methods, by which the mitigation hardware could be moved out to an approaching body, for either rendezvous or impact. Several possible combinations of mitigation technique and transportation option have been analyzed in detail.

Although there is much that still needs to be done, we would like to offer the following conclusions and recommendations.

\section{Public Awareness}

Despite the best efforts of Hollywood, the level of public awareness of this threat is still not high. Compared with other comparable threats, planetary body collision is still viewed as being a matter of science fiction rather than one of scientific fact.

While not advocating steps that could lead to hysteria and panic, we feel that the facts about this problem should be properly presented to the general public so as to raise public understanding of the threat and the ways in which it can be mitigated. Only when in full possession of the facts can the voting public make an informed decision about what steps should be taken.

\section{Statistical Problem}


The lack of attention given to this threat is in part this is due to a statistical problem. The chance of a significant-sized object striking the Earth is fairly low, such collisions might take place perhaps only once or twice per century. This has led to the danger being downgraded when compared with other threats to public safety - particularly those relating to acts of terrorism. However, the probability of an impact taking place cannot be considered in isolation; proper account must also be taken of the likely consequences of such a collision. Even the impact of a relatively small body would probably be very severe with fatalities in the millions, wide scale destruction and a recovery time possibly extending over decades.

To obtain a proper assessment of the danger, some appropriate parameter such as the expected number of fatalities over a period of, say, a decade or a century, must be considered. To illustrate this point, consider Table 7. It shows the chances of death by a variety of causes for a typical resident of the United States. It is interesting to note that the probability of dying due to a planetary body impact is about the same as that of dying due to an aircraft crash

Table 7 - Causes of Death and associated probabilities for a US resident ${ }^{13}$

\begin{tabular}{|c|c|}
\hline Cause of Death & Chance \\
\hline Motor vehicle accident & 1 in 100 \\
\hline Homicide & 1 in 300 \\
\hline Fire & 1 in 800 \\
\hline Firearms accident & 1 in 2,500 \\
\hline Electrocution & 1 in 5,000 \\
\hline Asteroid/comet impact & 1 in 20,000 \\
\hline Passenger aircraft crash & 1 in 20,000 \\
\hline Flood & 1 in 30,000 \\
\hline Tornado & 1 in 60,000 \\
\hline Venomous bite or sting & 1 in 100,000 \\
\hline Fireworks accident & 1 in $1,000,000$ \\
\hline Food poisoning by botulism & 1 in $3,000,000$ \\
\hline Drinking water with EPA limit of trichobethylene & 1 in $10,000,000$ \\
\hline
\end{tabular}

As Table 7 shows, this approach presents an altogether more worrying perspective on the danger.

3. Funding of Future Work

While a number of NEO search activities are currently underway, most are proceeding with very limited funding. In some countries, government agencies have declined to provide funding and - as we have recounted - actually poured scorn on the detection efforts. We strongly recommend that funding for these efforts be increased. In particular, sufficient high-quality instruments must be made available to conduct an all-latitude observation program with the aim of cataloging the entire NEO population.

Although funding is limited for NEO surveys and searches, at least it is non-zero. By comparison, the study of mitigation techniques is - with the notable exception of this present effort - almost totally unfunded. Equally important, research into new mitigation techniques is non-existent - except in those cases where the technology is under study for some other application.

We strongly recommend that a coherent study of mitigation techniques as well as their likely effectiveness, cost and deployment times, be undertaken in the very near future. This study, which would represent an enlarged follow-on to this work, should involve and call upon all of NASA's considerable resources, as 
well as those of the Department of Defense, the Armed Forces and other government agencies (e.g. the National Oceanic and Atmospheric Agency, the Federal Emergency Management Agency, etc.) International collaboration and funding should also be actively sought.

4. Development and Deployment of Mitigation Systems

The technical work undertaken in this study shows clearly that, although the mitigation challenge is formidable, it is not beyond our capabilities, provided preparations are begun well in advance of an impact. Despite the impression given by Hollywood, it is not practical to wait until a specific threat is identified before starting work on the mitigation system. Systems engineering, system deployment and - in some cases - technology development, will take several years.

We recommend most strongly that, following an appropriate study phase, a development program be initiated immediately, with a view to deploying an operational system as soon as possible. It is already clear to us that a first-generation protection system will not be able to counter all possible threats, however it should be able to defeat those most likely to occur. At the outset of Project Apollo, it was said that, while the United States could not guarantee to come first in the race to the Moon, failure to act would guarantee that she would come last. In the same way, it might be said that while we cannot guarantee success in protecting the Earth against a cosmic impact, failure to act will, in the long run, guarantee a major catastrophe of regional - if not global - proportions.

\section{Accomplishments}

It was not the intent of this study to select a particular technical option for recommendation as a threat mitigation system. Instead our intent was to study the various options, in several cases using improved and updated modeling techniques. It was also our aim to categorize these options into different mission configurations and to propose a method for comparing the large number of possible combinations of mitigation options and mission configurations. It was also our intent to recommend future work.

Several new tools were created during the course of this project. None should be regarded as a finished product and all would benefit from further development and refinement. As an example, the outbound trajectory tool is designed to give a first approximation of the required $\square \mathrm{V}$, using high thrust calculation methods. Similarly, the inbound tool takes a velocity vector at the point of impact and integrates the trajectory backward in time until the object is well outside the Earth's sphere of influence. It then integrates forward, after a deflection $\square \mathrm{V}$ has been applied to the object, so as to determine the resulting miss-distance from the Earth. The program iterates until a specified closest approach to the Earth has been achieved. Both these tools would benefit from the use of more accurate - although more calculationally intensive - techniques.

Numerous outbound propulsion systems and threat mitigation options were considered and modeled using several tools, which were created by combining some basic principles of physics with engineering data available in the open literature. These tools yielded first approximations for the performance and mass of each technical option.

We have built on the data and tools available in the literature to create a threat assessment tool that calculates the percentage of the total threat that can be defeated using a given mitigation system.

We have also created a procedure for comparing all these technologies in the future. In so doing we have identified mission categories for these technologies and have simplified future analyses by developing a procedure that deals with each category, instead of attempting to deal with each individual technology combination separately.

\section{Assessment of Mitigation Options}

Although it was not the purpose of this study to select mitigation options, a preliminary assessment is possible. Table 8 summarizes the capability of each major system option. 
The combination of a mass driver and a staged chemical outbound propulsion system offered good performance, but required long operating times to achieve the required deflection. Long operating times appear to be inherent whenever a mass driver is used. The total time required could have been improved somewhat by the use of a more advanced outbound propulsion technique. The $80-\mathrm{mT}$ mass driver design used here is not capable of deflecting even a 100-meter diameter asteroid within the overall time constraints imposed for the purposes of this study. Use of a larger mass driver, or a much linger operating time, would improve performance.

By comparison, the combination of a nuclear blast system and a staged chemical outbound propulsion system also offered excellent performance. Once again, it was the staged chemical propulsion system that limited the system performance.

A kinetic deflection vehicle, carried out by a staged chemical system, is theoretically capable of deflecting large asteroidal bodies. However, the interceptor vehicle mass required increases rapidly with asteroid size. Nonetheless, deflection of a 100 -meter diameter asteroid is possible.

The nuclear pulse option performs well on account of its use of the same - very effective - technique for both outbound travel and deflection. Of all the options considered during this study, nuclear pulse offers the best prospect of providing an effective mitigation technique using existing and near-term technology.

The solar collector system showed itself to be capable, but only at the expense of very large sail area and the consequent operational problems. As with the nuclear pulse option, it has the advantage of using a single unified system for both outbound propulsion and deflection.

Each of these options may well find some application in the future, but our initial results indicate that the nuclear pulse option offers the best defensive capabilities in the near term. This is by no means a recommendation but merely an observation based on the data at hand. Due to level of fidelity and extensive assumptions we have been forced to make in this limited study we strongly recommend that all options discussed here as well as other options suggested elsewhere be carried forward into a higher fidelity analysis

Future Work

A large amount of future work has been identified. All of our tools would benefit from more detailed analysis procedures. Many of the assumptions made during the development of our technology tools are in need of refinement. Our trajectory tools would benefit greatly from the ability to model continuous thrust propulsion systems. Our threat-assessment tool requires more research into the available data on the asteroid and comet population. As a minor example of this, we note that there are suggestions in the literature that cometary rings, such as the Leonid ring, may have non-uniform densities along their circumference. Since the Earth passes through such rings on a yearly basis, there would be a synodic period on which the Earth would cross these higher density areas, yielding a higher probability of impact. Our threat-assessment tool also requires further research into the consequences of an impact.

There are several other mitigation options that were not studied because of resource and time limitations; two of these are of particular interest and merit some mention. The first is laser ablation, used as either a remote station or as a rendezvous option. This technique would allow deflection in a manner similar to that of the solar collector, with a beam of high-energy, coherent light being directed at the incoming asteroid or comet. The second new option involves firing inert masses from a mass driver located in Earth orbit (perhaps at a Lagrange Point). This would combine our mass driver and kinetic deflection/fragmentation tools and would represent another remote station option.

Finally, we need to establish a method to combine the quantitative results from this analysis, and the qualitative issues outlined for each technology in the outbound propulsion and threat mitigation sections, in order to compare architectures. 
After completion of the more advanced tools above, including the atmospheric re-entry tool described earlier for fragmentation options, we could begin the overall threat assessment flowchart as described in Figure 13.

\section{Summary Conclusion}

We believe that the threat posed by NEO's should be taken very seriously. We also believe that it is well within humanity's ability to effectively defend itself against this threat. Development of the necessary technologies would also offer considerable synergy with NASA's other missions aimed at understanding the universe and exploring space. The planetary defense mission is also one for which NASA is uniquely suited and could potentially offer the Agency a goal that both fires the public imagination and also creates a sense of urgency comparable to that during the Apollo program in the 1960's. We shall endeavor to persuade those in positions of authority to continue the efforts presented here.

Table 8 - Summation of Parametric Results for Mitigation Concepts

\begin{tabular}{|l|c|c|c|c|c|}
\hline & \multicolumn{1}{|c|}{$\begin{array}{c}\text { Time Before } \\
\text { Impact (days)/ } \\
\text { Outbound } \\
\text { Travel Time } \\
\text { (days) }\end{array}$} & $\begin{array}{c}\text { Total System Mass at } \\
\text { SOI (m) for Different } \\
\text { Asteroid Diameters } \\
\text { (meters) }\end{array}$ & $\begin{array}{c}\text { Maximum } \\
\text { (meters)/Total } \\
\text { System Mass } \\
\text { at Earth SOI } \\
\text { (mT) }\end{array}$ \\
\hline System
\end{tabular}




\section{ACKNOWLEDGEMENTS}

We would like to thank our LaRC management and the RASC activity for funding this worthwhile endeavor, and the MSFC astronomy group for the technical assistance of Roy Young and the MSFC flight projects group for the assistance of Jon Campbell.

\footnotetext{
${ }^{1}$ Adams, R. B., Alexander, R., Bonometti, J., Chapman, J., Fincher, S., Hopkins, R., Kalkstein, M., Polsgrove, T., Statham, G., White, S., Survey of Technologies Relevant to Defense from Near Earth Objects, NASA-TP, to be published fall 2003.

${ }^{2}$ French, Bevan M., Traces of Catastrophe, Lunar and Planetary Institute, 1998

${ }^{3}$ Adushkin, Vitaly V. and Nemchinov, Ivan V., "Consequences of Impacts of Cosmic Bodies on the Surface of the Earth", Hazards Due to Comets and Asteroids, p721-778, The University of Arizona Press, Tucson, 1994

${ }^{4}$ Harvard-Smithsonian Minor Planet Center, http://cfa-www.harvard.edu/iau/Animations/Inner.gif

${ }^{5}$ Bate, R. R., Mueller, D. D., White, J. E. Fundamentals of Astrodynamics. New York, Dover Publications, Inc., 1971.

${ }^{6}$ Chapman, C. R, and Morrison, D., "Impacts on the Earth by Asteroids and Comets: Assessing the Hazard," Nature, 6 January 1994.

${ }^{7}$ Gold, R. E., "SHIELD - A Comprehensive Earth Protection System: A Phase I Report to the NASA Institute for Advanced Concepts," 28 May 1999.

${ }^{8}$ Lewis, John S., Comet and Asteroid Impact Hazards on a Populated Earth, Academic Press, 1999.

9 Jeffers, S. V., Manley, S. P., Bailey, M. E., and Asher, D. J., "Near-Earth Object Velocity Distributions and Consequences for the Chicxulub Impactor," Mon. Not. R. Astron. Soc., 327 (2001).

${ }^{10}$ Chesley, S, Chodas, P, Milani,, A., Valsecchi, G., Yeomans. D., "Quantifying the risk posed by potential Earth impacts," Icarus Asteroids, 2001.

${ }^{1}$ Ivezic, Zeljko, et al, "Solar System Objects Observed in the Sloan Digital Sky Survey Commissioning Data," The Astronomical Journal, November 2001.

${ }_{12}$ Shoemaker, E. M., "Asteroid and Comet Bombardment of the Earth," Annual Review of Earth and Planetary Sciences, 11: 461-494.

${ }^{13}$ Chapman, C.R. \& Morrison, D., 1994, Nature 367, 33-40
} 